\title{
Politique
}

Politique

Pierre BRUNEAU, Les villes moyennes au Québec. Leur place dans le système socio-spatial, Sillery, Presses de l'Université du Québec, 1989,195 p.

\section{Serge Belley}

Numéro 17, hiver 1990

Les nouveaux enjeux du politique

URI : https://id.erudit.org/iderudit/040657ar

DOI : https://doi.org/10.7202/040657ar

Aller au sommaire du numéro

Éditeur(s)

Société québécoise de science politique

ISSN

0711-608X (imprimé)

1918-6584 (numérique)

Découvrir la revue

Citer ce compte rendu

Belley, S. (1990). Compte rendu de [Pierre BRUNEAU, Les villes moyennes au Québec. Leur place dans le système socio-spatial, Sillery, Presses de l'Université du Québec, 1989,195 p.] Politique, (17), 172-175.

https://doi.org/10.7202/040657ar

Ce document est protégé par la loi sur le droit d'auteur. L'utilisation des services d'Érudit (y compris la reproduction) est assujettie à sa politique d'utilisation que vous pouvez consulter en ligne.

https://apropos.erudit.org/fr/usagers/politique-dutilisation/
Cet article est diffusé et préservé par Érudit.

Érudit est un consortium interuniversitaire sans but lucratif composé de l’Université de Montréal, l'Université Laval et l'Université du Québec à Montréal. Il a pour mission la promotion et la valorisation de la recherche. https://www.erudit.org/fr/ 
Pierre BRUNEAU, Les villes moyennes au Québec. Leur place dans le système socio-spatial, Sillery, Presses de l'Université du Québec, 1989, 195 p.

Les ouvrages sur les villes moyennes au Québec ne sont pas légion. Aussi, à l'heure de la mondialisation des échanges et face à l'intégration économique croissante des villes à l'échelle nationale voire internationale, on ne pourra que féliciter l'auteur de s'être intéressé au devenir des villes moyennes. Préciser la place et le rôle de ces dernières dans le système social-spatial québécois en regard des liens qu'elles entretiennent avec les autres villes et surtout les grandes métropoles, voilà l'essentiel de la tâche à laquelle s'est attaqué Pierre Bruneau dans ce livre.

L'approche privilégiée par l'auteur, qui est géographe de formation, repose, on s'en doutera, sur les théories et les méthodes de la géographie, mais fait aussi appel à certains concepts couramment utilisés en économie régionale, en sociologie et en science politique. En rapport avec cette dernière, mentionnons seulement le recours aux concepts d'État, de pouvoir, de domination et de régulation sur lesquels repose une bonne partie du cadre théorique et méthodologique utilisé par l'auteur. Pour cette raison, les politologues spécialistes des questions locales et régionales trouveront un certain intérêt à lire ce livre, cependant qu'ils n'y apprendront pas beaucoup de choses nouvelles sur les rapports de l'État à l'espace.

Dans la première partie de l'ouvrage dont le titre annonce qu'il sera question du contexte de développement des villes moyennes dans les pays industrialisés, l'auteur définit d'abord (chapitre 1) les principaux concepts (hiérarchie urbaine, intégration et domination) et expose les propositions théoriques qui orientent sa démarche. Parmi celles-ci, on retiendra celle voulant que «l'intégration croissante des villes au système urbain national voire international, favorise les changements de taille, le franchissement de seuils et, partant, la hiérarchisation qui provoque à son tour la spécialisation des niveaux urbains intermédiaires et inférieurs» (p. 6). 
Partant de cette proposition générale, l'auteur aborde ensuite la réalité des villes moyennes au Québec. Se référant aux critères de taille et de rayonnement régional, il identifie au total dix-neuf agglomérations urbaines appartenant à la catégorie des villes moyennes au Québec. Celles-ci, qui couvrent l'essentiel du territoire québécois habité et dont la taille moyenne s'établit à 38000 habitants représentent, après Montréal, la catégorie de villes qui regroupe le plus grand nombre d'habitants. Ce sont par ailleurs elles qui, entre 1976 et 1982, ont enregistré la plus forte croissance démographique, soit $14,3 \%$ comparativement à $4,7 \%$ pour l'ensemble des villes.

En raison de leur dynamisme, et à la lumière de ce que nous enseignent le discours officiel et le contre-discours sur les villes moyennes en Europe (chapitre 2), il est à prévoir, estime Bruneau, que les villes moyennes seront appelées à jouer un rôle accru dans la nouvelle organisation spatio-économique qui prend forme au Québec. Le sort réservé aux villes moyennes représente donc un enjeu de taille, aussi bien du point de vue du redéploiement en cours ou à prévoir du système productif (dispersion industrielle), que de la régionalisation (décentralisation/déconcentration) des services publics.

On regrettera dans cette première partie, que l'auteur n'ait fait référence qu'au seul cas de la France, comme si le discours sur les villes moyennes dans ce pays était représentatif «du contexte de développement des villes moyennes dans les pays industrialisés».

Dans la deuxième partie de l'ouvrage, l'auteur aborde la question des villes moyennes au Québec sous l'angle du redéploiement industriel. Rappelant d'abord les fondements de la théorie des pôles de croissance (chapitre 4) et ensuite les contraintes nouvelles que fait peser sur les firmes industrielles et l'État l'internationalisation de la production (chapitre 5), l'auteur analyse ensuite les liens industriels que les villes moyennes québécoises entretiennent entre elles et avec les autres villes à l'échelle nationale et internationale (chapitre 6).

Parmi les 1540 établissements industriels recensés en 1983 dans les dix-neuf villes moyennes du Québec, 20\% d'entre eux, constate Bruneau, sont, en raison de leur statut juridique (suc- 
cursale, division ou filiale), commandés de l'extérieur. Une situation qui pèse d'autant plus lourdement sur le devenir de ces villes, que plus de $60 \%$ de leur main-d'oeuvre industrielle travaillent dans ces établissements. Dans $40 \%$ des cas, ces entreprises sont gérées à partir de Montréal, dans $12 \%$ des cas de Toronto et dans 7,5\% des cas des États-Unis. La montée récente de l'entrepreneuriat dans certaines régions, affirme Bruneau, ne va pas nécessairement à l'encontre de l'intégration industrielle croissante des villes moyennes aux grandes aires métropolitaines, mais laisse présager que l'initiative pourrait désormais appartenir, au moins partiellement, aux villes moyennes.

Sans aucun doute, ce dernier chapitre est de loin le plus intéressant et le plus stimulant, aussi bien sur le plan théorique qu'empirique. Une analyse plus approfondie de l'expérience des sommets socio-économiques régionaux permettrait ici d'étayer les conclusions de l'auteur.

Dans la dernière partie de l'ouvrage, l'auteur analyse la place et le rôle des villes moyennes sous l'angle du redéploiement du tertiaire public. En regard du redéploiement du tertiaire moteur (chapitre 8), Bruneau conclut que tant en ce qui concerne la diffusion que la production des connaissances (recherche industrielle et universitaire), les régions sont amenées à se spécialiser dans des créneaux d'enseignement et de recherche avantageux pour elles, mais s'inscrivant dans les priorités nationales. Il en va cependant différemment pour les grands centres urbains qui, bien que conduits eux aussi à se spécialiser dans certains secteurs d'excellence en raison de la mondialisation des marchés, monopolisent l'essentiel du savoir critique, c'est-à-dire de la recherche dite fondamentale. Ce qui fait dire à Bruneau que le redéploiement du tertiaire moteur s'effectue lui aussi dans le sens d'une intégration plus poussée des villes moyennes, diminuant d'autant leur autonomie par rapport aux grands centres.

Dans la nouvelle spatialité qui prend forme et qui détermine le rôle des villes moyennes au Québec, l'État, affirme Bruneau, n'est pas en reste (chapitre 9). Que ce soit par le biais de ses stratégies de décentralisation/déconcentration ou encore par la réforme territoriale amorcée au début des années 60 et qui se poursuit 
actuellement avec la mise en place des MRC, son objectif demeure le même. Il s'agit, selon lui, d'accroître, tout autant pour des raisons d'efficacité administrative que de réduction des coûts des services publics, son contrôle sur l'espace et par là, sur les populations, dans une optique de régulation de l'activité sociale. Dans la mise en oeuvre de ses stratégies de régulation, l'État peut désormais compter sur les élites régionales devenues, depuis peu, les «organisatrices de l'activité collective» en région.

On regrettera ici que la plus grande partie des documents gouvernementaux cités par l'auteur concerne presqu'exclusivement la période 1976-1984 (gouvernement péquiste), laissant ainsi dans l'ombre la période d'avant 1976 et surtout celle plus récente, marquée par le retour au pouvoir des libéraux à Québec et l'arrivée des conservateurs à Ottawa. La thèse de l'État-ordonnateur-deshommes-et-des-choses (sur le territoire) qui fonde ici la problématique de l'auteur, est en effet non seulement réductrice des changements politiques, mais également des rapports politiques souvent conflictuels entre les élus provinciaux et fédéraux d'une part, et entre ces derniers et les élites politiques régionales d'autre part.

En dépit de ces critiques, l'ouvrage de Pierre Bruneau représente une contribution fort valable qui vient alimenter la réflexion sur le développement et le devenir des villes moyennes au Québec.

Serge Belley Université Laval 